\title{
Is it time for biomarker-based diagnostic criteria for prodromal Alzheimer's disease?
}

\author{
Kaj Blennow* and Henrik Zetterberg
}

\begin{abstract}
Drug candidates targeting amyloid- $\beta$ (A $\beta$ ) pathology

in Alzheimer's disease are in different phases of

clinical trials. These treatments will probably be most

effective in the earlier stages of the disease, before

neurodegeneration is too severe, but at the same

time symptoms are vague and the clinical diagnosis

is difficult. Recent research advances have resulted in

promising biomarkers, including cerebrospinal fluid

analyses for tau and $A \beta$, magnetic resonance imaging

measurement of atrophy, and positron emission

tomography imaging of glucose metabolism and $A \beta$

pathology, which allow identification of prodromal

Alzheimer's disease. More details are needed, however,

on how these biomarkers can be standardized,

to allow a general implementation in the clinical

routine diagnostic work-up of patients with cognitive

disturbances.
\end{abstract}

It is now more than 25 years since the National Institute of Neurological and Communicative Disorders and Stroke and the Alzheimer's Disease and Related Disorders Association (now the Alzheimer's Association) established the most commonly used diagnostic criteria for Alzheimer's disease (AD) [1]. These criteria require that the patient is demented before a diagnosis of $\mathrm{AD}$ can be made. We now know, however, that dementia represents a late stage of $\mathrm{AD}$ and that $\mathrm{AD}$-specific neurodegeneration starts many years earlier. Disease-modifying drugs that attack primary pathogenic processes underlying $\mathrm{AD}$ will probably be most effective in the earlier stages of the disease, before plaque and tangle load and neurodegeneration have become too severe.

\footnotetext{
*Correspondence: kaj.blennow@neuro.gu.se

Clinical Neurochemistry Laboratory, Institute of Neuroscience and Physiology, Department of Psychiatry and Neurochemistry, The Sahlgrenska Academy at University of Gothenburg, Sahlgrenska University Hospital, SE-431 80 Mölndal, Sweden
}

If we want to do something substantial about the prospects of $\mathrm{AD}$ patients in the near future, we need to develop diagnostic algorithms that recognize predementia stages of the disease. This has become possible thanks to brain imaging and cerebrospinal fluid (CSF) analyses that predict $\mathrm{AD}$ with dementia in patients with mild cognitive impairment [2-4]. Accordingly, new research criteria for the diagnosis have been proposed that capture both the prodromal and the more advanced dementia stages of the disease in the same diagnostic framework [5]. In the present article, we discuss what is needed before this type of criteria can be implemented in the clinical routine diagnostic work-up of patients with cognitive disturbances.

\section{Validated biomarkers for Alzheimer's disease}

CSF levels of total tau reflect cortical axonal degeneration, levels of phospho-tau reflect tangle pathology and levels of the 42 -amino-acid isoform of amyloid $\beta$ (A $\beta 42$ ) reflect brain amyloid pathology [2]. Magnetic resonance imaging (MRI) of hippocampal atrophy gauges progression of the neurodegeneration in a manner that correlates well with both neuropathological measures of tangle load and cognitive symptoms [3]. 2[18 F]-Fluoro-2-deoxy-Dglucose positron emission tomography (FDG-PET) allows for the assessment of the glucose metabolism rate in specific brain regions and can detect metabolic dysfunctions in brain regions affected by $\mathrm{AD}$ also in pre-dementia stages [4]. Amyloid plaques in the living human brain can be visualized using amyloid-binding PET tracers such as Pittsburgh Compound B (PIB) [4]. Marked PIB retention is found in $\mathrm{AD}$ patients in areas of the brain known to contain large amounts of $A \beta$ plaques.

\section{When do the biomarkers turn positive?}

In a recent review, Jack and coworkers condense the $\mathrm{AD}$ biomarker literature to model the sequence of pathological events in $\mathrm{AD}$ [6]. The first biomarker change to occur in $\mathrm{AD}$ is probably the lowering of the CSF A $\beta 42$, reflecting the formation of oligomers and loose aggregates of $A \beta$ in the brain. This change is followed by PIBPET positivity as a sign of accumulation of fibrillar $A \beta$ in the brain. $A \beta$ oligomerization, or an unknown molecular 
change, induces metabolic dysfunction - seen as lowered glucose metabolism on FDG-PET - and axonal degeneration and hyperphosphorylation of tau - seen as elevations in total tau and phospho-tau concentrations in the CSF. Eventually, the axonal and neuronal loss in specific brain regions is manifested as reduced MRI volumes of, for example, the hippocampus, which parallels cognitive decline. The model is supported by a vast body of literature and suggests that most biomarkers listed above are positive in the mild cognitive impairment stage of $\mathrm{AD}$ and that amyloid-related biomarkers are the most promising antecedent biomarkers for AD [6].

\section{What is the diagnostic performance of these biomarkers?}

Sensitivity and specificity figures for the different biomarkers vary between studies but tend to be above $80 \%$ for distinguishing $\mathrm{AD}$ patients from cognitive normal controls and for identifying incipient AD in the mild cognitive impairment stage of the disease, at least in standardized monocenter studies [2-4]. Only a few of the biomarkers have as yet undergone testing in large multicenter settings.

\section{Why are Alzheimer's disease biomarkers not $100 \%$ specific?}

Currently available biomarkers do not show full-proof diagnostic accuracy. Except for technical shortcomings with the biomarkers, however, there are several fundamental reasons why $100 \%$ sensitive and specific biomarkers for $\mathrm{AD}$ are an unreachable goal. Most biomarker studies are based on clinically diagnosed cases, which introduces a relatively large percentage of misdiagnosis [7]. There is also a large overlap in pathology between AD and other dementias, such as Lewy body dementia and vascular dementia $[8,9]$. This overlap in pathology essentially precludes the possibility of finding biomarkers that have close to $100 \%$ sensitivity and specificity for AD. One way out of this conundrum might be to reconsider the terminology. Perhaps we should stop using the term AD biomarkers and instead acknowledge that the biomarkers reflect distinct pathogenic or pathologic processes; for example, amyloid retention in the brain and degeneration of nonmyelinated cortical axons. These changes, especially in combination, are frequently seen in $\mathrm{AD}$ but may also be present in other neurodegenerative disorders, especially in isolation.

It is known that a significant percentage of nondemented older people have enough plaques and tangles to warrant a neuropathological diagnosis of AD [10]. Recent biomarker studies corroborate this finding. For example, a relatively large portion of cognitively normal older people have CSF A $\beta 42$ values or PIB-PET binding similar to that found in AD cases [2-4]. These findings raise the question of whether the term normal aging has to be redefined. Longitudinal studies will tell us whether these individuals do have preclinical AD, or whether silent AD pathology is part of the normal aging process.

\section{Which biomarkers should be used and how?}

More detailed guidelines on how biomarkers should be implemented in the diagnostic procedure for early $\mathrm{AD}$ are needed for them to be used in clinical routine. Notably, these guidelines have to be down-to-earth to allow for general implementation. A large number of unresolved issues need to be settled.

\section{Assessment of memory}

There is to date no consensus on which tests should be used to identify impairment in episodic memory and other cognitive domains beyond what is expected due to normal aging [11]. Further, a major challenge will be to determine cut-off points that can be generally applied to identify patients with cognitive dysfunction, since a cutoff point set in one population does not necessarily apply to populations with other ethnic or socioeconomic characteristics.

Biochemical markers in the cerebrospinal fluid

Although assays for measurement of tau and A $\beta$ in CSF have been well validated and the biological variability for these biomarkers is low [2], there is a variation in biomarker levels in reports from different research centers, even when using the same assay [12]. Moreover, there is no accepted gold standard method and no consensus on what assays are to be used. A first study comparing CSF biomarker levels between laboratories found excellent within-laboratory variation, but a large variation between centers [13]. Similar results were reported in a large multicenter study [14]. This variation complicates multicenter research studies and trials, and also precludes the introduction of generally applicable cut-off levels. To help deal with this problem, a global quality control program for CSF biomarkers was recently launched [12].

\section{Structural magnetic resonance imaging}

The utility of structural imaging in diagnostic guidelines will be increased by standardization of acquisition and analysis methods [3]. Which is the region of interest with regards to AD-type brain atrophy measured on MRI? Which methods and what cut-off points should be used to distinguish age-related brain atrophy from atrophy in $\mathrm{AD}$ ?

\section{Positron emission tomography}

Similar standardization issues that apply to MRI also apply to PET imaging of cerebral $A \beta$ aggregation and glucose metabolism. Where in the brain should amyloid retention be quantified using amyloid PET? Which of the 
different radioligands to label amyloid plaques should be used? Where in the brain should we monitor cerebral glucose metabolism using FDG-PET, and should we focus on different regions in different disease stages?

\section{How about availability and costs?}

For the implementation of these biomarkers in a diagnostic algorithm, availability and financial considerations may be of importance. Amyloid PET is at present only available in highly specialized centers. In Sweden, the approximate cost is $\$ 200$ for CSF biomarkers (combined analysis of total tau, phospho-tau and A 342 ), $\$ 500$ for structural MRI and \$5,000 for amyloid PET figures that probably vary considerably between countries.

\section{How should we move forward?}

There is an enormous amount of literature showing good or excellent diagnostic performance of several biomarkers reflecting different facets of the disease process in $\mathrm{AD}$. We have unprecedented possibilities to phenotype our patients. We think the time is ripe to boldly develop the biomarker-based research criteria proposed by Dubois and coworkers [5] into a detailed, practical and feasible diagnostic algorithm that will be applicable in clinics worldwide. It is easy to predict that this will be a challenging process. The proposed algorithm would require evaluation in a longitudinal clinical multicenter study of patients with memory problems to assess its diagnostic (or strictly speaking as it is now, predictive) accuracy against postconversion clinical dementia diagnoses and, whenever possible, to neuropathological findings before general implementation in the clinic. Such an evaluation will require some significant period of time. It is in the best interest of all who wish to develop better therapeutic paradigms for this devastating disease that this process be started now.

\section{Abbreviations}

$A \beta$, amyloid $\beta ; A D$, Alzheimer's disease; CSF, cerebrospinal fluid; FDG, $2\left[{ }^{18} \mathrm{~F}\right]$ fluoro-2-deoxy-D-glucose; $M R I$, magnetic resonance imaging; $P E T$, positron emission tomography; PIB, Pittsburgh Compound B.

\section{Competing interests}

KB has served on an advisory board for Innogenetics, Belgium. The authors declare no other competing interests.

\section{Acknowledgements}

Work in the authors' laboratories is supported by the Swedish Research Council, the Royal Swedish Academy of Sciences and the Alzheimer's Association
Published: 30 April 2010

\section{References}

1. McKhann G, Drachman D, Folstein M, Katzman R, Price D, Stadlan EM: Clinical diagnosis of Alzheimer's disease: report of the NINCDS-ADRDA Work Group under the auspices of Department of Health and Human Services Task Force on Alzheimer's Disease. Neurology 1984, 34:939-944.

2. Blennow K, Hampel H, Weiner M, Zetterberg H: Cerebrospinal fluid and plasma biomarkers in Alzheimer disease. Nat Rev Neurol 2010, 6:131-144.

3. Frisoni GB, Fox NC, Jack CR, Jr, Scheltens P, Thompson PM: The clinical use of structural MRI in Alzheimer disease. Nat Rev Neurol 2010, 6:67-77.

4. Nordberg A, Rinne JO, Kadir A, Langstrom B: The use of PET in Alzheimer disease. Nat Rev Neurol 2010, 6:78-87.

5. Dubois B, Feldman HH, Jacova C, Dekosky ST, Barberger-Gateau P, Cummings J, Delacourte A, Galasko D, Gauthier S, Jicha G, Meguro K, O'Brien J, Pasquier F, Robert P, Rossor M, Salloway S, Stern Y, Visser PJ, Scheltens P: Research criteria for the diagnosis of Alzheimer's disease: revising the NINCDS-ADRDA criteria. Lancet Neurol 2007, 6:734-746.

6. Jack CR Jr, Knopman DS, Jagust WJ, Shaw LM, Aisen PS, Weiner MW, Petersen RC, Trojanowski JQ: Hypothetical model of dynamic biomarkers of the Alzheimer's pathological cascade. Lancet Neurol 2010, 9:119-128.

7. Engelborghs S, De Vreese K, Van de Casteele T, Vanderstichele H, Van Everbroeck B, Cras P, Martin JJ, Vanmechelen E, De Deyn PP: Diagnostic performance of a CSF-biomarker panel in autopsy-confirmed dementia. Neurobiol Aging 2008, 29:1143-1159.

8. Kotzbauer PT, Trojanowsk JQ, Lee VM: Lewy body pathology in Alzheimer's disease. J Mol Neurosci 2001, 17:225-232.

9. Schneider JA, Arvanitakis Z, Leurgans SE, Bennett DA: The neuropathology of probable Alzheimer disease and mild cognitive impairment. Ann Neurol 2009, 66:200-208.

10. Price $J$, Morris JC: Tangles and plaques in nondemented aging and 'preclinical' Alzheimer's disease. Ann Neurol 1999, 45:358-368.

11. Black R, Greenberg B, Ryan JM, Posner H, Seeburger J, Amatniek J, Resnick M, Mohs R, Miller DS, Saumier D, Carrillo MC, Stern Y: Scales as outcome measures for Alzheimer's disease. Alzheimers Dement 2009, 5:324-339.

12. Mattsson N, Blennow K, Zetterberg H: Inter-laboratory variations in CSF biomarkers for Alzheimer's disease: united we stand, divided we fall. Clin Chem Lab Med 2010, in press. [Epub ahead of print]

13. Verwey NA, van der Flier WM, Blennow K, Clark C, Sokolow S, De Deyn PP, Galasko D, Hampel H, Hartmann T, Kapaki E, Lannfelt L, Mehta PD, Parnetti L, Petzold A, Pirttila T, Saleh L, Skinningsrud A, Swieten JC, Verbeek MM, Wiltfang J, Younkin S, Scheltens P, Blankenstein MA: A worldwide multicentre comparison of assays for cerebrospinal fluid biomarkers in Alzheimer's disease. Ann Clin Biochem 2009, 46:235-240.

14. Mattsson N, Zetterberg H, Hansson O, Andreasen N, Parnetti L, Jonsson M, Herukka SK, van der Flier WM, Blankenstein MA, Ewers M, Rich K, Kaiser E, Verbeek M, Tsolaki M, Mulugeta E, Rosén E, Aarsland D, Visser PJ, Schröder J, Marcusson J, de Leon M, Hampel H, Scheltens P, Pirttilä T, Wallin A, Jönhagen ME, Minthon L, Winblad B, Blennow K: CSF biomarkers and incipient Alzheimer disease in patients with mild cognitive impairment. JAMA 2009, 302:385-393.

doi:10.1186/alzrt31

Cite this article as: Blennow K, Zetterberg $\mathrm{H}$ : Is it time for biomarker-based diagnostic criteria for prodromal Alzheimer's disease? Alzheimer's Research \& Therapy 2010, 2:8. 